\title{
The Radar Ambiguity Function Application for the Frequency Modulated Signals Designing
}

\author{
A. Kawalec, C. ZioŁkowski, C. Leśnik* and J. Pietrasinski \\ Military University of Technology, Gen. S. Kaliskiego 2, 00-908 Warszawa, Poland
}

\begin{abstract}
The optimization of the transmitted signal time-frequency structure is the very important part of the radar system design from the point of view of the radar system effectiveness. In this meaning the radar ambiguity function is the basic tool used for the radar system features testing. The synthesis procedure of the signal with simultaneous amplitude and phase manipulation is presented in the paper. The procedure is based on the Zak transform that is connected with the radar ambiguity function. The Legendre polynomials were applied in the procedure mentioned above. The results of the simulation tests are presented as examples of the consideration main effects.
\end{abstract}

PACS numbers: 84.40.Ua, 84.40.Xb

\section{Introduction}

The radar transmit signal with linear frequency modulation (LFM) is very common in practice. It results from its advantages from the radar applications point of view. Despite the LFM signal importance there is necessity to look for new types of transmitting signals giving a chance to improve the quality of information taken from radar systems. Moreover, there is possibility to reach such the ambitious goals thanks to among others technology development concerning for example solid state transmitters etc. As a result, signal generation with amplitude modulation becomes possible. $>$ From these reasons to look for more sophisticated methods of the radar transmit signals synthesis is reasonable. His paper is concentrated on one of them.

The synthesis method of the radar transmitted signal with amplitude and frequency modulation is considered in the paper. It is based on the relation between the signal radar ambiguity function (RAF) and the signal Zak transform. According to literature $[1-6]$ the Chebyshev polynomials are applied in this case. In the paper Legendre polynomials are used. The second part of the paper consists of the problem formulation and general description of the signal synthesis method based on the relation between the signal RAF and the signal Zak transform. Analytic description of the synthesis method based on the Legendre polynomials is presented in Sect. 3 of the paper. The problem solution for the 8th Legendre polynomial in a shape of simulation tests results concerning synthesized signal is described in Sect. 4. Short conclusions are written in Sect. 5.

* corresponding author; e-mail: Czeslaw.Lesnik@wel.wat.edu.pl

\section{Problem formulation}

Radar transmitted signals with the amplitude, frequency or phase manipulation is on of the very important signal family $[7,8]$. The signal, consisting of the subpulses sequence with the unitary duration time and with the amplitude and frequency manipulation can be described by the formula as follows:

$$
f(t)=\sum_{m=-M}^{\mathrm{M}} \sum_{n=-N}^{\mathrm{N}} a_{m n} \operatorname{rect}(t-m) \exp (\mathrm{j} 2 \pi n t)
$$

where $a_{m n}-$ elements of the matrix $A=$ $\left[a_{m n}\right]_{(2 M+1) \times(2 N+1)}$ that represents sub-pulses series coefficients, $m$ - the sub-pulse number, $n$ - the number of the frequency component being $n$-th multiple of the unitary frequency step in the $m$-th sub-pulse and

$$
\operatorname{rect}[t-m]= \begin{cases}1 & \text { for }-0.5+m \leq t<0.5+m \\ 0 & \text { for elsewhere } t\end{cases}
$$

The problem can be formulated as follows: having the matrix $\boldsymbol{A}$ one should match values of its elements and its dimension in order to achieve the time-frequency signal structure that assures feature expected from the radar tasks point of view. In order to find solution the RAF was applied that describes the signal $f(t)$ complex envelope at the optimum receiver output as a function of the delay time $\tau$ and the Doppler frequency shift $f_{\mathrm{d}}$. It is described by the formula as follows:

$$
\chi_{f}\left(\tau, f_{\mathrm{d}}\right)=\int_{-\infty}^{\infty} f(t) f^{*}(t+\tau) \exp \left(-\mathrm{j} 2 \pi f_{\mathrm{d}} t\right) \mathrm{d} t .
$$

In order to find the RAF of the signal (1) the Zak transform was used that is defined as follows [1]: Let us assume that the function $f \in L^{2}(\boldsymbol{R})$ is known. The Zak transform $Z_{f}\{f(t)\}$ of the function $f(t)$ is called the such 
representation in which $Z_{f}: f \rightarrow Z_{f}\{f\} \in L_{1}^{2}\left(\boldsymbol{R}^{2}\right)$ and $\forall x, y \in\langle 0,1\rangle$ it is truth that

$$
Z_{f}(x, y)=\sum_{k=-\infty}^{\infty} f(x+k) \exp (-\mathrm{j} 2 \pi k y) .
$$

One can show that between the signal RAF and its Zak transform there is relation described by the dependence as follows [1]:

$$
\begin{aligned}
& \chi_{f}\left(\tau, f_{\mathrm{d}}\right)=\int_{0}^{1} \int_{0}^{1}\left|Z_{f}(x, y)\right|^{2} \\
& \quad \times \exp \left(-\mathrm{j} 2 \pi\left(f_{\mathrm{d}} x+\tau y\right)\right) \mathrm{d} x \mathrm{~d} y, \quad \tau, f_{\mathrm{d}} \in \boldsymbol{Z} .
\end{aligned}
$$

Based on (4) and assuming the integrand [1] periodic feature one can conclude that the RAF values in the integer points $\left(\tau, f_{\mathrm{d}}\right)$ represent the periodic function $\left|Z_{f}(x, y)\right|^{2}$ expansion coefficients into two-dimensional exponential Fourier series.

As a result of the Zak transform of the signal defined by Eq. (1) and using theorem concerning linearity and shift in the time domain one can show that [1]:

$$
\begin{aligned}
& Z_{f}(x, y)=\sum_{m=-M}^{M} \sum_{n=-N}^{N} a_{m n} \exp (\mathrm{j} 2 \pi(n x-m y)) \\
& \quad=P(x, y),
\end{aligned}
$$

where $P(x, y)$ is a function of two variables $x$ and $y$. This function is periodic from the reason the both variables have the period equal to 1 . One can conclude that according to (5) it is visible that

$$
\left|Z_{f}(x, y)\right|^{2}=|P(x, y)|^{2} \text {. }
$$

Based on (4) and (6) it can be written that

$$
\begin{aligned}
& \chi_{f}\left(\tau, f_{\mathrm{d}}\right)=\int_{0}^{1} \int_{0}^{1}|P(x, y)|^{2} \\
& \quad \times \exp \left(-\mathrm{j} 2 \pi\left(f_{\mathrm{d}} x-\tau y\right)\right) \mathrm{d} x \mathrm{~d} y, \quad \tau, f_{\mathrm{d}} \in \boldsymbol{Z} .
\end{aligned}
$$

Based on (7) one can conclude that the signal (1) RAF values for integer values $\left(\tau, f_{\mathrm{d}}\right)$ are the periodic function $|P(x, y)|^{2}$ expansion coefficients into two-dimensional exponential Fourier series. The matrix $\boldsymbol{A}$ of the coefficients of the assumed signal model corresponds with the discrete values of the RAF described by (7). Based on (5) the matrix $\boldsymbol{A}$ elements can be used as the expansion coefficients of the function $P(x, y)$ into two-dimensional exponential Fourier's series. It means that

$$
a_{m n}=\int_{0}^{1} \int_{0}^{1} P(x, y) \exp (-\mathrm{j} 2 \pi(n x-m y)) \mathrm{d} x \mathrm{~d} y .
$$

Taking all together to describe a periodic function $P(x, y)$ is needed. It should make able both to calculate the RAF $\chi_{f}\left(\tau, f_{\mathrm{d}}\right)_{\tau=m, f_{\mathrm{d}}=n}$ values and to find coefficients $a_{m n}$ of the designed signal structure. A general scheme of the analysed problem solution is as follows:

$$
\begin{aligned}
& \underset{x, y \in\langle 0,1\rangle}{P(x, y)} \stackrel{|\cdot|^{2}}{\longrightarrow}|P(x, y)|^{2} \stackrel{F}{\longrightarrow} \mid \underset{\tau=m, f_{\mathrm{d}}=n}{\chi_{f}\left(\tau, f_{\mathrm{d}}\right) \mid}\left\{\begin{array}{l}
\text { acceptable } \\
\text { features }
\end{array}\right\} \\
& \quad \underset{x, y \in\langle 0,1\rangle}{P(x, y)} \stackrel{F}{\longrightarrow} a_{m n} .
\end{aligned}
$$

There are two possibilities concerning the periodic function $P(x, y)$ description in the case of the synthe- sis of the signal with simultaneous manipulation in the amplitude and frequency. One of them is the function $P(x, y)$ formulation based on the Chebyshev polynomials of the first kind and $k$ order [1]. In this paper to apply the Legendre polynomials of the first kind and $k$ order in the signal synthesis procedure is assumed.

\section{Radar signal synthesis using Legendre polynomials}

Mentioned above expectations concerning the function $P(x, y)$ fulfils the period function based on the Legendre polynomials $P_{k}(z)$ of the first kind and $k$ order. The polynomial of order both 0 and 1 are respectively as follows: $P_{0}(z)=1, P_{1}(z)=z$. The others can be determined from the recurrent formula

$$
P_{k+1}(z)=\frac{2 k+1}{k+1} z P_{k}(z)-\frac{k}{k+1} P_{k-1}(z) .
$$

In the considered case the polynomial is the function of two variables $z=\varphi(x, y)$. Let us take into account the formulae (7) and (8) that are basic for these method and take into account that the Legendre polynomials values for arguments from the real numbers set belong to the real numbers set, too. As a result one can obtain

$$
\begin{aligned}
& \chi_{f}\left(\tau, f_{\mathrm{d}}\right)=\int_{0}^{1} \int_{0}^{1} P_{k}^{2}[\varphi(x, y)] \\
& \quad \times \exp \left(-\mathrm{j} 2 \pi\left(f_{\mathrm{d}} x-\tau y\right)\right) \mathrm{d} x \mathrm{~d} y, \quad \tau, f_{\mathrm{d}} \in \boldsymbol{Z}, \\
& a_{m n}=\int_{0}^{1} \int_{0}^{1} P_{k}[\varphi(x, y)] \\
& \quad \times \exp (-\mathrm{j} 2 \pi(n x-m y)) \mathrm{d} x \mathrm{~d} y .
\end{aligned}
$$

Taking into consideration the trigonometric function

$$
z=\varphi(x, y)=\cos 2 \pi \sqrt{x^{2}+y^{2}}
$$

as the $z=\varphi(x, y)$ representation, the RAF of the synthesized signal describes the formula as follows:

$$
\begin{aligned}
& \chi_{f}\left(\tau, f_{\mathrm{d}}\right)=\int_{0}^{1} \int_{0}^{1} P_{k}^{2} \cos \left(2 \pi \sqrt{x^{2}+y^{2}}\right) \\
& \quad \times \exp \left(-\mathrm{j} 2 \pi\left(f_{\mathrm{d}} x+\tau y\right)\right) \mathrm{d} x \mathrm{~d} y, \quad \tau, f_{\mathrm{d}} \in \boldsymbol{Z} .
\end{aligned}
$$

In the next step the coefficients $a_{m n}$ values are found according to (15) under condition that the RAF described according to (14) fulfils expectations. Then

$$
\begin{gathered}
a_{m n}=\int_{0}^{1} \int_{0}^{1} P_{k} \cos \left(2 \pi \sqrt{x^{2}+y^{2}}\right) \\
\quad \times \exp (-\mathrm{j} 2 \pi(n x-m y)) \mathrm{d} x \mathrm{~d} y .
\end{gathered}
$$

For further consideration it was assumed that analogously to [1] the number of the matrix $\boldsymbol{A}$ significant elements is $2 k+1$ and that each row in the matrix consists of only one dominant element. It means that for each sub-pulse of the designed radar pulse is dedicated exactly to one frequency. Taking into account above comments it can be stated that the expected radar signal structure that makes able to achieve the RAF requested parameters is as follows: 


$$
s(t)=\sum_{m=-k}^{k} a_{m n_{m x}} \operatorname{rect}(t-m) \exp \left(\mathrm{j} 2 \pi n_{m x} t\right),
$$

where

$$
\begin{aligned}
& a_{m n_{m x}}=\max _{n \in\{-k, \ldots, k\}}\left\{a_{m n}\right\} \\
& \text { and } \quad n_{m x}: \underset{n \in\{-k, \ldots, k\}}{\forall} a_{m n} \leq a_{m n_{m x}} .
\end{aligned}
$$

\section{Simulation results}

For this consideration explanation it was assumed that the Legendre polynomial order is $k=8$. On the basis of the iterative expression (10) one can achieve

$$
\begin{aligned}
& P_{8}(z)=\frac{1}{128}\left(6435 z^{8}-12012 z^{6}+6930 z^{4}\right. \\
& \left.-1260 z^{2}+35\right) .
\end{aligned}
$$

Taking into account expression (13) and trigonometric identity for cosine function even powers the expression (17) has a shape as follows:

$$
\begin{aligned}
& P_{8}\left(\cos 2 \pi \sqrt{x^{2}+y^{2}}\right)=\frac{1}{2048} \\
& \times\left[\frac{6435}{8} \cos \left(16 \pi \sqrt{x^{2}+y^{2}}\right)\right. \\
& +429 \cos \left(12 \pi \sqrt{x^{2}+y^{2}}\right) \\
& +\frac{693}{2} \cos \left(8 \pi \sqrt{x^{2}+y^{2}}\right) \\
& \left.+315 \cos \left(4 \pi \sqrt{x^{2}+y^{2}}\right)+\frac{1225}{8}\right] .
\end{aligned}
$$

Assuming that for $k=8$ the RAF described on the base of expression (14) fulfils expectations, in the next step the coefficients $a_{m n}$ values of the radar signal wanted structure were found. These coefficients were calculated according to the expression as follows:

$$
\begin{aligned}
& a_{m n}=\frac{1}{2048} \int_{0}^{1} \int_{0}^{1}\left[\frac{6435}{8} \cos \left(16 \pi \sqrt{x^{2}+y^{2}}\right)\right. \\
& +429 \cos \left(12 \pi \sqrt{x^{2}+y^{2}}\right) \\
& +\frac{693}{2} \cos \left(8 \pi \sqrt{x^{2}+y^{2}}\right)+315 \cos \left(4 \pi \sqrt{x^{2}+y^{2}}\right) \\
& \left.+\frac{1225}{8}\right] \exp (-\mathrm{j} 2 \pi(n x-m y)) \mathrm{d} x \mathrm{~d} y .
\end{aligned}
$$

The time-causal shape of the wanted signal was described with the help of the expression as follows:

$$
s(t)=\sum_{m=0}^{16} a_{m n_{m x}} \operatorname{rect}(t-m-0.5) \exp \left(\mathrm{j} 2 \pi n_{m x} t\right),
$$

where

$$
\begin{aligned}
& a_{m n_{m x}}=\max _{n \in\{-8, \ldots, 8\}}\left\{a_{m n}\right\} \\
& \text { and } n_{m x}: \underset{n \in\{-8, \ldots, 8\}}{\forall} a_{m n} \leq a_{m n_{m x}} .
\end{aligned}
$$

The RAF (to be more precise - the radar ambiguity surface) designed with the help of (14) is shown in Fig. 1. It was done on the integer lattice for the Legendre polynomial with $k=8$. The analogous RAF but described with the help of the Chebyshev polynomial with $k=8$ is shown in Fig. 2 for comparison. One can notice the higher side lobes concentration around the main lobe in the case of the Legendre polynomial case with relation to the Chebyshev polynomial case.

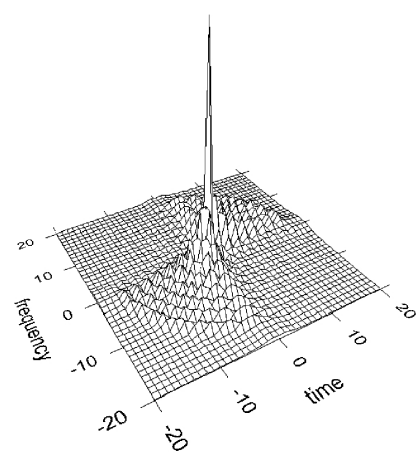

Fig. 1. Ambiguity surface on the integer lattice for Legendre polynomial with $k=8$.

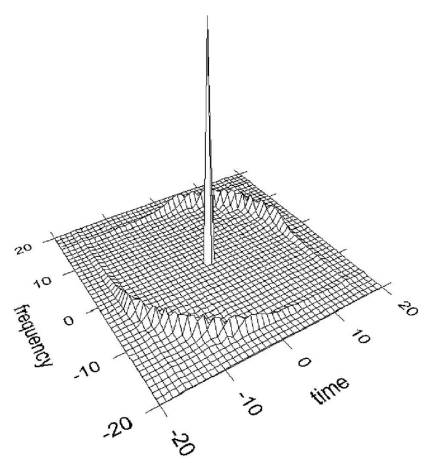

Fig. 2. Ambiguity surface on the integer lattice for Chebyshev polynomial with $k=8$.

The coefficients matrixes $\boldsymbol{A}$ described thanks to (21) for the Legendre and Chebyshev polynomials are shown in Figs. 3 and 4, respectively.

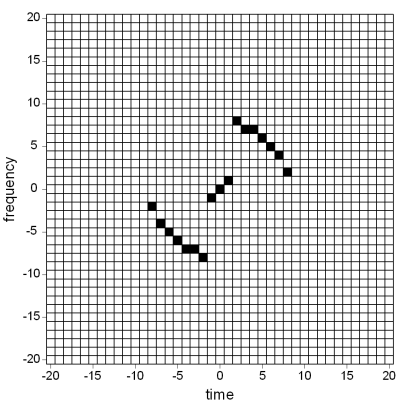

Fig. 3. Matrix $\boldsymbol{A}$ for Legendre polynomial with $k=8$.

The simulation research results confirm previous assumption according to which in each row of the matrix $\boldsymbol{A}$ there is only one significant element. 


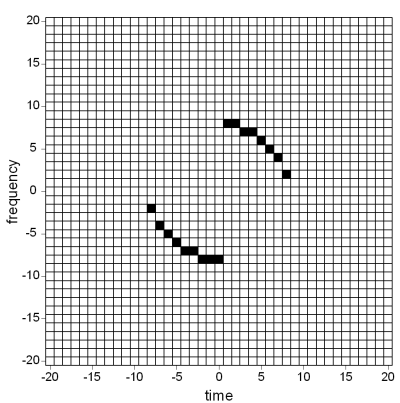

Fig. 4. Matrix $\boldsymbol{A}$ for Chebyshev polynomial with $k=8$.

The synthesized signal real and imaginary part for the Legendre and Chebyshev polynomial respectively are shown in Figs. 5 and 6.

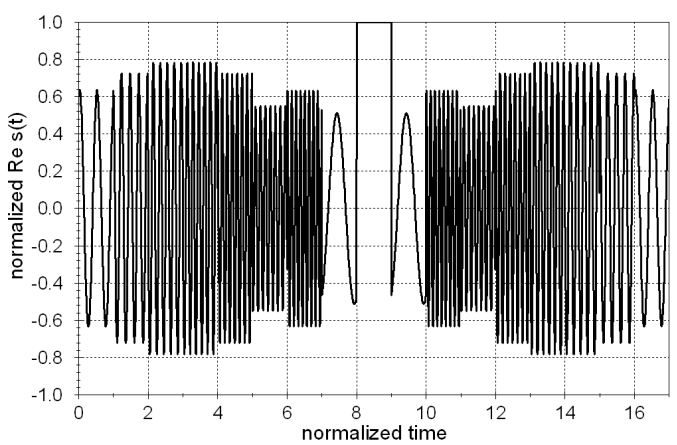

Fig. 5. Real and imaginary part of the $s(t)$ signal for Legendre polynomial with $k=8$.

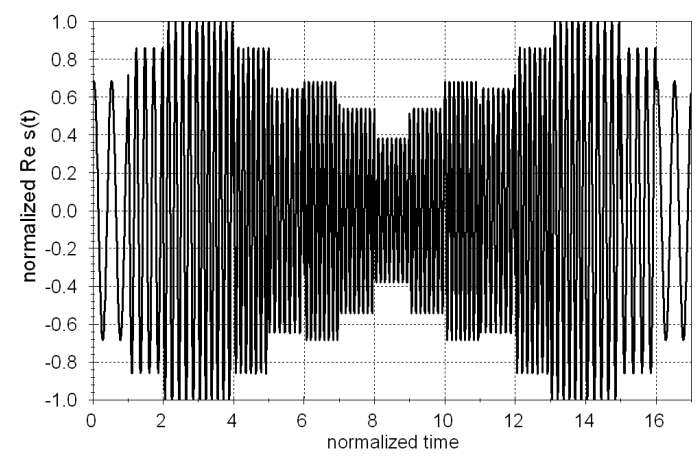

Fig. 6. Real and imaginary part of the $s(t)$ signal for Chebyshev polynomial with $k=8$.

$>$ From the presented figures it results that according to assumptions the synthesized signal is manipulated both in frequency and in amplitude. The signal synthesized with the help of the Legendre polynomial consists of the sub-pulses having zero frequency that corresponds to the carrier frequency of the real signal as opposite to the Chebyshev polynomial case.

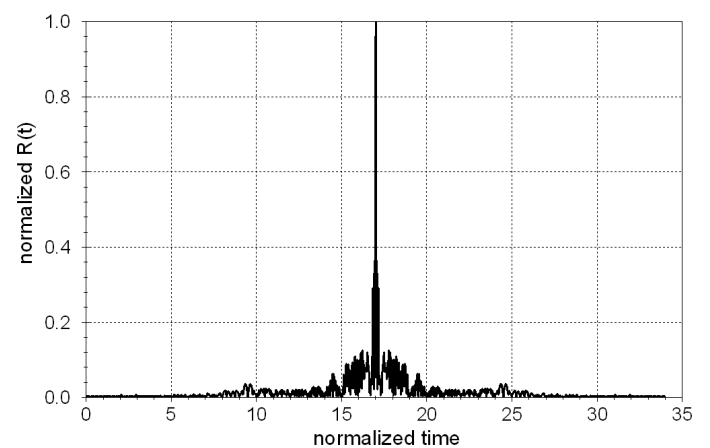

Fig. 7. Autocorrelation function of the $s(t)$ signal for Legendre polynomial with $k=8$.

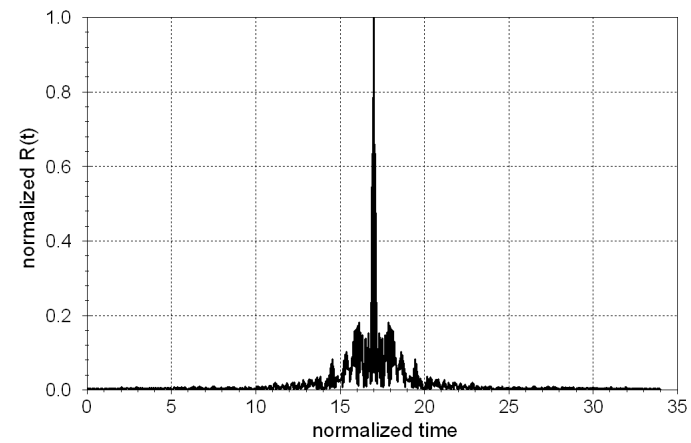

Fig. 8. Autocorrelation function of the $s(t)$ for Chebyshev polynomial with $k=8$.

For the synthesized signal its autocorrelation function was described. This function represents the signal time shape at the matched filter output in the case of the zero Doppler frequency shift. The autocorrelation function of the signals synthesized with the help of the Legendre and Chebyshev polynomials are shown in Figs. 7 and 8, respectively.

The side lobes level of the autocorrelation function of the signal synthesized based on the Legendre polynomial is about $3 \mathrm{~dB}$ lower than it is in the case of the Chebyshev polynomials application with approximately the same main lobe width.

\section{Summary}

The Legendre polynomials usefulness for radar signal synthesis with amplitude and frequency manipulation was shown in the paper. The side lobe levels of the signal aperiodic autocorrelation function for the polynomials with $k=8$ reach values about $3 \mathrm{~dB}$ lower with comparison with the results concerning the Chebyshev polynomials application with the same order. The side lobes distribution on the $\tau f_{\mathrm{d}}$ plane is different for the Legendre and Chebyshev polynomials application. In the case of the Legendre polynomials one can notice the higher side lobes concentration around the main lobe of the RAF. 
The disadvantages of the Legendre polynomials are their complicated analytic description in comparison with the Chebyshev polynomials. It makes considerations more complicated especially for the higher order of the polynomials.

The paper is the kind of proof that looking for the wider class of polynomials used in radar signal synthesis is reasonable.

\section{Acknowledgments}

This work was supported by the National Centre for Research and Development for the years 2007-2010 under Commissioned Research Project PBZ-MNiSWDBO-04/I/2007.

\section{References}

[1] I. Gladkova, IEEE Trans. Aerospace Electron. Syst. 37, 4 (2001).

[2] A. Szpakowski, C. Tyszkiewicz, T. Pustelny, Acta Phys. Pol. A 114, A-237 (2008).

[3] T. Pustelny, A. Opilski, A. Pustelny, Acta Phys. Pol. A 114, A-183 (2008).

[4] A. Snakowska, Mol. Quant Acoust. 28, 241 (2007).

[5] P. Kaniewski, Mol. Quant. Acoust. 28, 151 (2007).

[6] P. Kaniewski, Mol. Quant. Acoust. 28, 165 (2007).

[7] Ch.E. Cook, M. Bernfeld, Radar Signals. An Introduction to Theory and Application, Artech House, Inc., Boston 1993.

[8] N. Levanon, E. Mozeson, Radar Signals, Wiley, Hoboken, New Jersey 2004. 